\title{
Surgical Treatments on Patients with Anterior Cervical Hyperostosis-Derived Dysphagia
}

\author{
Ah Rom Song, M.D., Hee Seung Yang, M.D., Eunjin Byun, M.D., Youngbae Kim, M.D. ', \\ Kwan Ho Park, M.D. ${ }^{2}$, Kyung Lyul Kim, M.D. ${ }^{3}$ \\ Departments of Rehabilitation Medicine, ${ }^{1}$ Orthopedic Surgery, ${ }^{2}$ Neurosurgery, Seoul Veterans Hospital, Seoul 134-791, \\ ${ }^{3}$ Ahn Ui Health Center, Hamyang-gun 676-823, Korea
}

Anterior cervical hyperostosis may be a cause of dysphagia. For anterior cervical hyperostosis, medical or surgical treatments can be adhibited in view of the causative mechanisms and intensities of dysphagia. We report 3 cases of cervical hyperostosis-derived progressive dysphagia that underwent operation. Radiologic diagnosis and Video Fluoroscopic Swallowing Study were performed on the three patients for evaluation. One had history of recurrent aspiration pneumonia accompanied by weight loss, another complained of dysphagia only when swallowing pills, and the third experienced recurrence symptom with reossification. All patients reported gradual improvement of dysphagia immediately after their cervical osteophytes were resected through the anterior approach. In relation to postoperative improvement, however, they expressed different degrees of satisfaction according to severity of symptoms. Surgical treatment, performed for the anterior cervical hyperostosis-derived dysphagia, can immediately relieve symptoms of difficulty in swallowing. This might especially be considered as an appropriate treatment option for severe dysphagia.

Key Words Dysphagia, Hyperostosis, Surgical procedure

\section{INTRODUCTION}

Dysphagia refers to a difficulty in the whole or part of swallowing. This disorder occurs in the oral, pharyngeal and esophageal phases, thereby leading to a disorder of function where food in the oral cavity is transferred to

Received August 19, 2011; Accepted October 18, 2011

Corresponding author: Hee Seung Yang

Department of Rehabilitation Medicine, Seoul Veterans Hospital, 6-2, Doonchun-dong, Gangdong-gu, Seoul 134-791, Korea

Tel: +82-2-2225-1498, Fax: +82-2-2225-1579, E-mail: yang7310@naver. com

(c) This is an open-access article distributed under the terms of the Creative Commons Attribution Non-Commercial License (http:// creativecommons.org/licenses/by-nc/3.0) which permits unrestricted noncommercial use, distribution, and reproduction in any medium, provided the original work is properly cited.

Copyright ( 2012 by Korean Academy of Rehabilitation Medicine the gastrointestinal tract. In severe cases, it may cause complications, including aspiration pneumonia, bronchospasm, dehydration, malnutrition, and suffocation. ${ }^{1}$ Causes of dysphagia are diverse. According to Utsinger et al., ${ }^{2}$ it is known that among symptoms arising from cervical osteophytosis, dysphagia is developed at a ratio of $17 \%$. The anterior cervical osteophytosis is observed even in $20-30 \%$ among healthy people ${ }^{3}$ and may be a direct cause of dysphagia. ${ }^{4}$ Excessive osteophytosis of the cervical vertebrae may cause dysphasia via mechanisms, such as (1) a mechanical pressure on esophagus, (2) inflammation and edema on periphery, (3) cricopharyngeal spasm, and (4) abnormal recurvation of epiglottis. ${ }^{3,5}$ The therapeutic approaches considered for dysphagia include medication to reduce inflammation and edema around the cervical vertebrae, and a surgical treatment 
to restore the movement of epiglottis by correcting the anatomical compression and deformity of cervical vertebrae. ${ }^{3}$ Because of the side effects and mortality of surgical treatments, however, there is skepticism in using surgical treatments. Hence, we conducted a study on the effects of surgical treatments for patients with dysphagia caused by the anterior cervical osteophytes through 3 patient cases. One patient underwent surgical removal of the osteophytes because of severe weight loss from malnutrition and repetitive aspiration pneumonia, even though the patient had received conservative treatment for dysphagia by anterior cervical osteophyte. A second patient underwent surgical removal of the osteophyte in the early stage for mild dysphagia symptoms. The third patient needed a re-operation because of dysphagia caused by reossification found in the long term follow-up period even though the patient had undergone surgical removal of the anterior cervical osteophyte.

\section{CASE REPORTS}

\section{Case 1}

A 75-year-old male patient visited the hospital complaining of dysphagia and weight loss (as much as $5 \mathrm{~kg}$ ) that had occurred 3 months prior to this visit. Twenty years earlier, he had a received craniotomy on the left frontal parietal region because of traumatic subdural hemorrhage from falling and had recovered without sequelae. He had taken drug treatment for hypertension, but has no difficulty in daily living activity. For his dysphagia, he complained of more difficulties in swallowing solid foods rather than liquid foods and this was gradually progressing. Seven months after the onset of dysphagia, he was hospitalized, at which time he could only take small amounts of liquid foods instead of regular meals and his weight loss during 10 months since the onset of dysphagia was approximately $10 \mathrm{~kg}$. The patient did not complain of speech disturbance or dyspnea and both movements of the tongue and gag reflex were normal. On his brain MRI scan, partial encephalomalacia was observed on the lesion where the left frontal parietal craniotomy had performed and left inferior temporal gyrus; this was not different compared with a prior brain MRI scan, and any additional lesions which might induce dysphagia was not observed. An endoscopic examination was performed with suspicion of a pharyngolaryngeal tumor, but no abnormal findings were detected. There were also no abnormal results in routine blood and tumor marker tests. As excessive osteophytosis on the anterior part of the cervical vertebrae from $\mathrm{C}^{\text {rd }}$ to $\mathrm{C} 6{ }^{\text {th }}$ levels were observed in the cervical plain radiograph, a 3D cervical CT was performed and the identified posterior part of the pharyngolaryngeal cavity was compressed and narrowed by osteophytes from $\mathrm{C} 2{ }^{\text {nd }}$ to $\mathrm{C} 7^{\text {th }}$ vertebrae levels (Fig. 1). As the dysphagia by anterior cervical osteophytes was suspected, a VFSS (Video Fluroscopic Swallowing Study) was performed (Fig. 2). Findings revealed that a movement of the epiglottis displayed an abnormal recurvation by osteophytes, with at least $50 \%$ of food remaining in the epiglottic valleculae. Pyriform sinuses were observed in all tested materials, and asymptomatic aspiration was shown as 8 points of the PAS (Penetration-Aspiration Scale) in $2 \mathrm{cc}$ of diluted barium swallowing test. After the above examinations, an electronic stimulator, VitalStim ${ }^{\circledR}$ (Chaltanooga group, Austin, USA), was conducted one hour a day for 10 days. The treatment sessions were com-
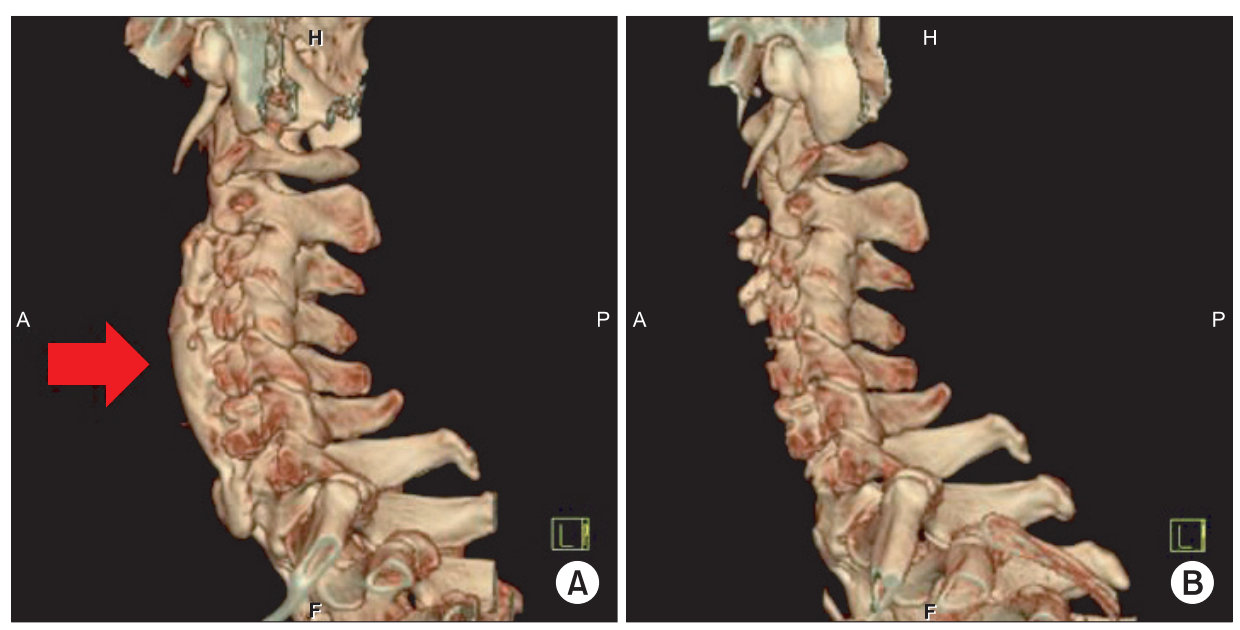

Fig. 1. Three-dimensional reconstruction computed tomography of cervical spine: anterior cervical osteophytes are revealed from C2 to $\mathrm{C} 7$ levels with the $\mathrm{C} 4$ level being the most prominent (A). Post-anterior approach of the cervical spine osteophytes resection at $\mathrm{C} 2$ to $\mathrm{C} 7$ levels (B). 
pleted in two weeks. The electronic stimulation used a 2-channel form with an alternating current, wherein one of the electrical pads of the first channel was attached between the musculus biventer mandibulae muscle and hyoid bone and another electrical pad was attached between the hyoid bone and thyroid cartilage. The electrical pad of the second channel was attached between the thyroid and cricoid cartilages and vertically to the inferior part of the cricoid cartilage. A week after completing the electronic stimulation treatment, a VFSS was performed, where it was found that aspiration and nasal regurgitation had disappeared and the remaining amount of food shown in the epiglottic valleculae and pyriform sinuses were reduced to below $10 \%$. Thus, it was possible to change his diet from liquid form to regular meals. After one month, however, dysphagia began to progress again and aspiration pneumonia had developed, which required hospitalization. Compared with previous findings, VFSS results revealed an increase in the amount of retention of contrast in the epiglottic valleculae and pyriform sinuses by $10-50 \%$ and the movement of epiglottis had decreased in all tested materials. It was suggested that his symptoms has worsened compared to previous findings. Due to persistent dysphagia, his diet was changed back to liquid food. He also received consultation in our depart- ment of neurosurgery for operation due to severe general weakness caused by insufficient nutrition. Next, he decided to have an operation after 2 year 7 months since the onset of dysphagia. Osteophytes cervical vertebrae from $\mathrm{C} 2{ }^{\text {nd }}$ to $\mathrm{C} 7^{\text {th }}$ levels were removed via an anterior approach (Fig. 1). After the operation, he recovered without any side effects and 1 month later, VFSS was performed. Based on the nasal reflux that had been observed in the swallowing water test disappeared and food remaining in the epiglottic valleculae and pyriform sinuses were below $10 \%$. In spite of the formation of the inner wall membrane, the remaining food was removed by chin tuck and repetitive swallowing. It was determined that the epiglottic motion had improved and the remainder in the epiglottic valleculae and pyriform sinus had reduced remarkably (Fig. 2). The patient was able to return to a general diet, had gained approximately $6 \mathrm{~kg}$ of weight, and was satisfied with his postoperative improvement of symptoms in spite of having subjective dysphagia for waterless biscuits.

\section{Case 2}

A 62 year-old male patient visited our department of neurosurgery, complaining of dysphagia and limited movement range of the cervical vertebrae that has oc-
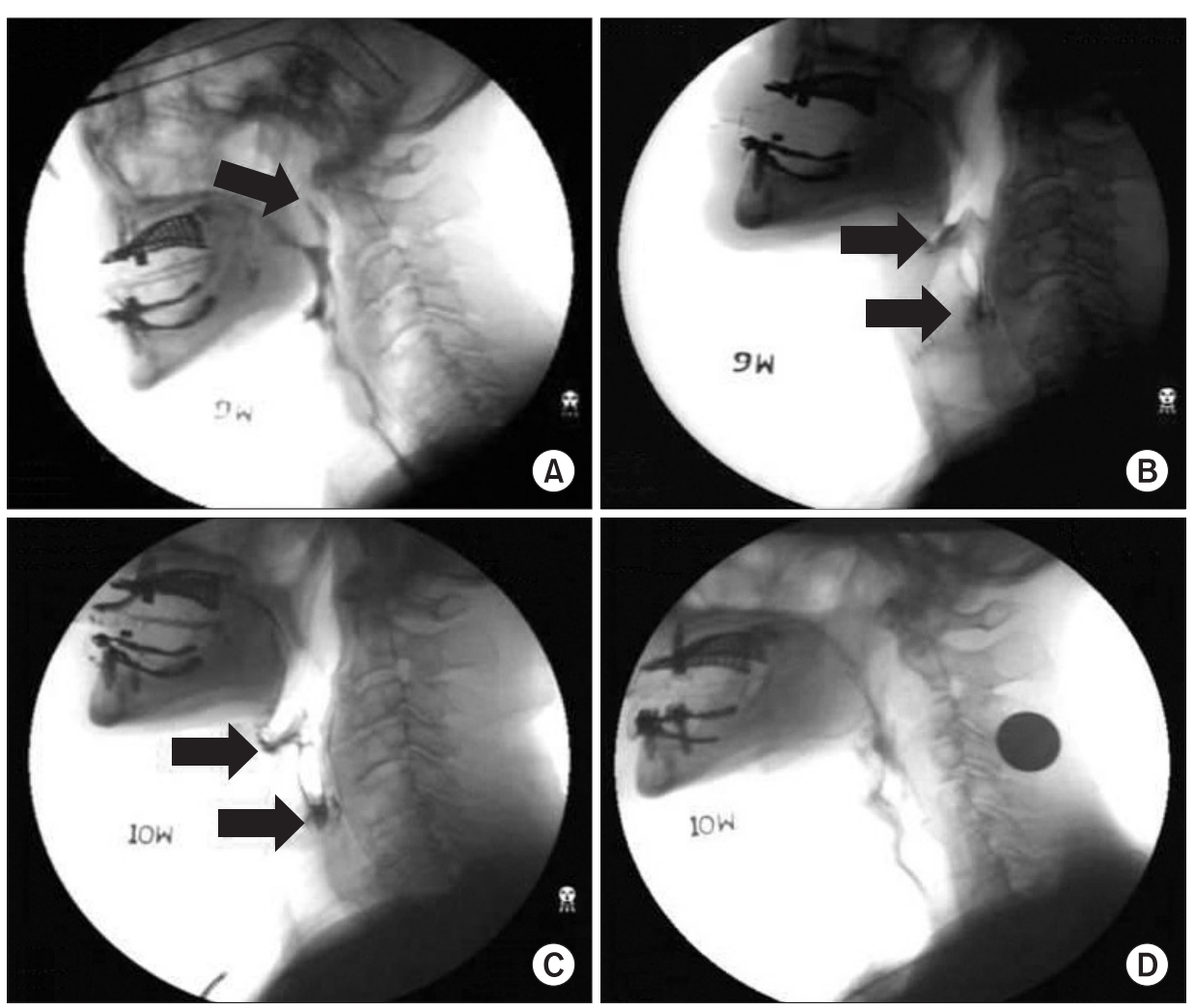

Fig. 2. The first video fluroscopic swallowing study (VFSS) showed a nasal regurgitation of contrast (A). After completing ten sessions of neuromuscular electrical stimulation (VitalStim $^{\circledR}$ ), the nasal regurgitation vanished. However, VFSS still showed minimal amount of retention of contrast in the valleculae and pyriform sinuses (B). 7 months later, the patient was suffering from an exacerbated symptom of difficulty in swallowing. A $10 \mathrm{cc}$ water swallowing test showed moderate amount of retention of contrast in the valleculae and pyriform sinuses (C). After removing the anterior cervical osteophytes, retention of contrast in the valleculae and pyriform sinuses was not evident at follow-up VFSS (D). 
curred for 7 months. He was diagnosed as having hypertension 3 years before and receiving medication. He felt no discomfort when taking a meal, but complained of difficulties in swallowing a tablet. There was no language disorder or dyspnea. During a physical examination, both motion and gag reflux of his tongue were normal. As an imaging test, a 3D cervical CT was performed and the posterior part of the pharyngolaryngeal cavity was compressed by an anterior bony spur of the $3^{\text {rd }}-6^{\text {th }}$ cervical vertebrae (Fig. 3). VFSS findings had determined that there was a remainder of $10-50 \%$ in the epiglottic valleculae and pyriform sinuses due to motor abnormality of the cricopharyngeal muscle caused by an anterior bony spur on the cervical vertebrae with the height of upper esophageal sphincter in swallowing cookies. In a swallowing test involving $10 \mathrm{cc}$ of water, vocal cord penetration was shown, but it was removed spontaneously (Fig. 3). The bony spur on the $4^{\text {th }}-6^{\text {th }}$ cervical vertebrae with anterior approach was removed. VFSS findings performed 2 weeks after the operation suggested that there was no remarkable change in the remainder of the epiglottic valleculae that was shown in swallowing cookies, but the remainder of the pyriform sinuses decreased dramatically, and the phenomenon of vocal cord penetration that had appeared during the water swallowing test also disappeared (Fig. 3). The patient reported a reduction in the number of feeling difficulties in taking tablet after surgery, but a complete improvement of the symptoms was not shown.

\section{Case 3}

After complaining of dysphagia, a 61-year-old male patient was operated under the diagnosis of diffuse idiopathic hyperostosis on the $2^{\text {nd }}-5^{\text {th }}$ cervical vertebra, and had since experienced symptoms improvement. However, his dysphagia began to progress again to being able to swallow only liquid food since approximately May, 2010. He was hospitalized in our department of orthopedics with $170 \mathrm{~cm}$ height, $46.8 \mathrm{~kg}$ of weight, and 16.18 of BMI. His medical history was unremarkable. In imaging tests performed after hospitalization, diffuse idiopathic hyperostosis on the $1^{\text {st }}-3^{\text {rd }}$ cervical vertebrae and anterior bony spur ranging from the $2^{\text {nd }}$ cervical vertebrae to the $1^{\text {st }}$ thoracic vertebrae were found (Fig. 4). VFSS findings showed that there was normal swallowing reflex in the
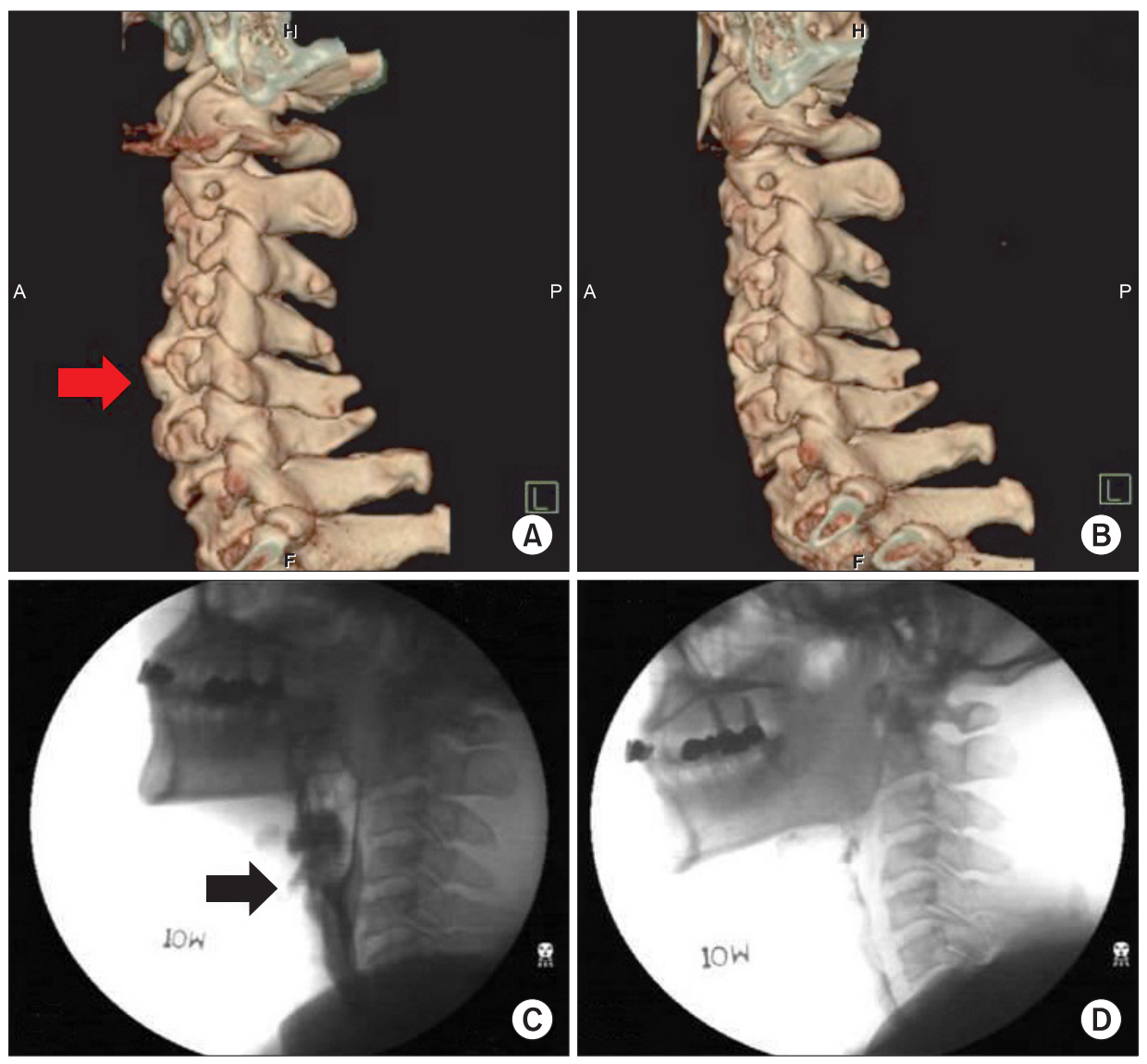

Fig. 3. Three-dimensional reconstruction computed tomography of the cervical spine revealed anterior cervical osteophytes from C4 to C6 levels (A). Post-anterior approach of the cervical spine osteophytes resection at C4-5-6 levels (B). Pre-operative video fluroscopic swallowing study (VFSS) showed the presence of laryngeal penetration in $10 \mathrm{cc}$ water swallowing test (C). After removing the anterior cervical osteophytes, laryngeal penetration was not detected at follow-up VFSS (D). 
pharyngeal phase, but because of an anterior bony spur on the $2^{\text {nd }}-4^{\text {th }}$ cervical vertebrae, there was an epiglottic recurvation disorder. There was also a remainder of $10-50 \%$ in the pyriform sinuses and with an increase of sample viscosity, not only did the amount of remainder in the pyriform sinuses increase but his complaint for the feeling of sticking in his throat got similarly worse. In addition, asymptomatic aspiration was observed in $5 \mathrm{cc}$ of a diluted barium test (Fig. 4). Next, there was removal of the osteophyte on the $2^{\text {nd }}, 3^{\text {rd }}, 4^{\text {th }}$, and $5^{\text {th }}$ cervical vertebrae discectomy, and fixation of the $3^{\text {rd }}$ and $4^{\text {th }}$ cervical vertebrae with an anterior approach for the improvement of dysphagia (Fig. 4). One month after the operation, VFSS findings determined that no aspiration was observed, the amount of remainder in the pyriform sinuses had decreased, but the amount of remainder increased with an increase of sample viscosity. Sinse abnormal movement of the epiglottis still remained, a 10-time application of VitalStim ${ }^{\circledR}$ was performed. After electric stimulation treatment, VFSS findings determined that the amount of remainder in the pyriform sinuses after swallowing was reduced and aspiration that had previously been observed had disappeared. He was able to have regular meals and improvement on symptoms of dysphagia increased by $80 \%$ compared with preoperative conditions.

\section{DISCUSSION}

Causes resulting in an anterior cervical bony spur include: 1) ankylosing spondylitis; 2) diffuse idiopathic skeletal hyperosteosis (DISH) causing ossification of the anterior longitudinal ligament and tendon; and 3) degenerative intervertebral disc inflammation or intervertebral osteochondrosis. ${ }^{6}$ The most common symptom caused by an anterior cervical bony spur is the worsening of dysphagia in consuming solid food. Approximately $28 \%$ of patients with the anterior cervical bony spur complain
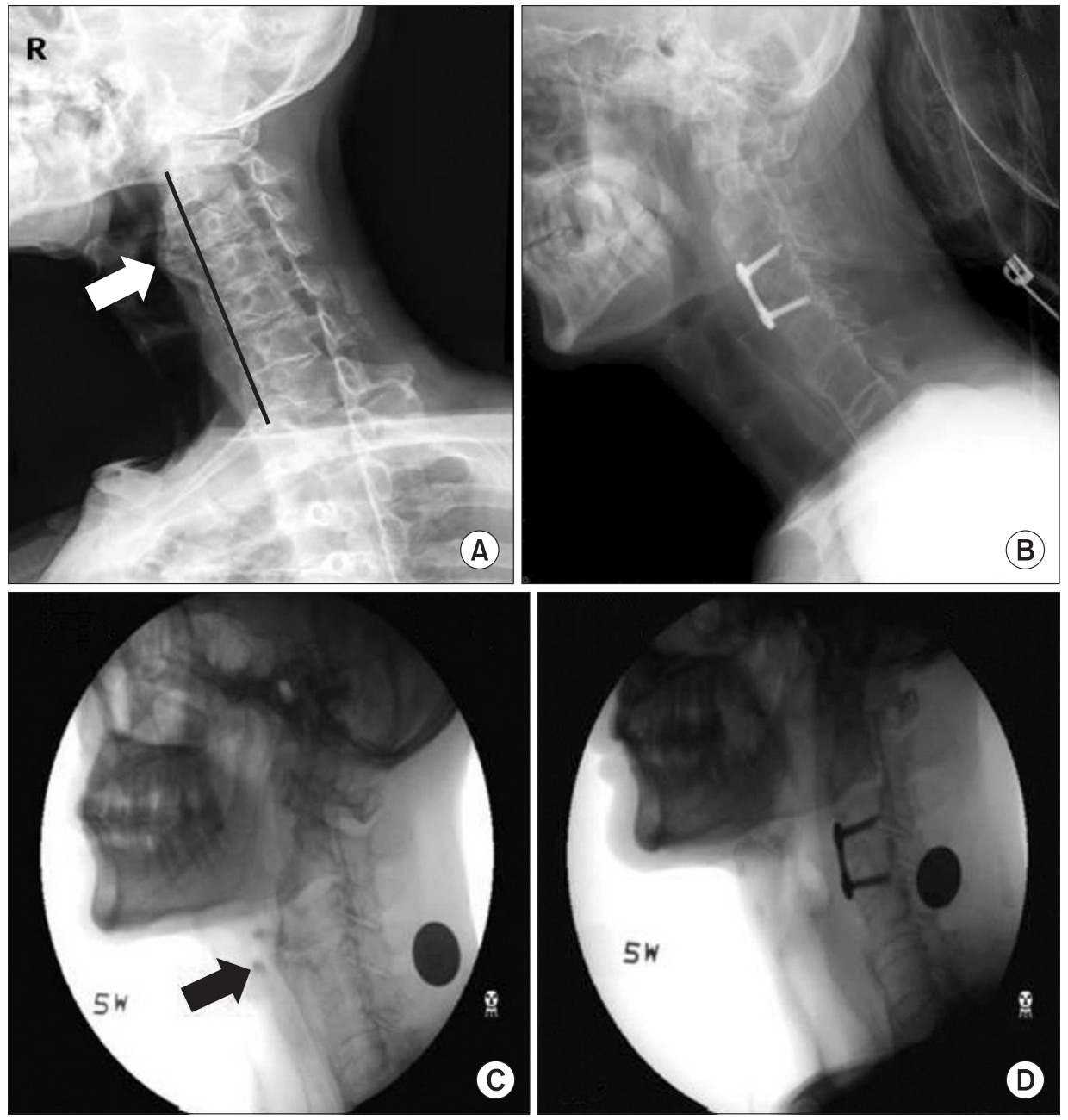

Fig. 4. The lateral view of cervical $\mathrm{X}$-ray revealed anterior cervical osteophytes at C2 and C3 levels (A). Post-anterior approach of the cervical spine osteophytes resection at C2-3-4-5 levels (B). Pre-operative video fluroscopic swallowing study (VFSS) showed the presence of laryngeal penetration in a $5 \mathrm{cc}$ water swallowing test (C). After removing the anterior cervical osteophytes, laryngeal penetration and retention of contrast in pyriform sinuses were not detected at follow-up VFSS (D). 
of dysphagia, $4 \%$ of which will visit a hospital with such a complaint. ${ }^{6,7}$ Dysphagia due to cervical osteophytosis is mainly developed from a mechanical obstruction of cricoid cartilage, secondary inflammation and edema. This results in pains and muscular spasm, as well as diseases, such as tumor in the hypopharynx, larynx, esophagus, vertebrae, lung, and mediastrinum, esophagitis, esophageal motor disease, esophageal stricture, pharyngoesophageal diverticulum, neuromuscular disease, cricopharyngeal rigidity, gastroesophageal reflux, stroke, and globus hystericus. ${ }^{6}$ Therefore, it is important to perform a full medical history, neurological, and physical examination, as well as a blood test, endoscopic test, radiological test, and VFSS when patients complain of dysphagia. Treatment by cervical hyperostosis should be decided depending on pathogenesis of dysphagia, its severity, and conditions. A preferred treatment is to take anti-inflammatory agent or steroids to reduce edema or inflammation of the pharynx, bronchus, larynx, and esophagus regions. ${ }^{3}$ Where there is no improvement even after drug medication and rehabilitation treatment and general weakness continues to progress from severe weight loss or continued aspiration pneumonia as shown in the patient of case 1, surgical treatment should be considered. This surgical treatment removes the mechanical compression of esophagus, and in most cases, there is an improvement of symptoms. ${ }^{3}$ However, there are certain cases, such as the patient of case 3 , where dysphagia reoccurs from reossification in long term follow-up even though the bony spur on the anterior cervical vertebra causing dysphagia had been removed. Miyamoto et al. ${ }^{10}$ has identified that for all the patients who had their bony spur on the anterior cervical vertebra removed, reossification developed and growth rate of the bony spur was $1 \mathrm{~mm}$ a year on average. The side effects of bony spur removal of the anterior cervical vertebrae include recurrent neuropalsy, hematoma, infection, and fistula formation. ${ }^{5}$ There is therefore skepticism in the successful surgical treatment of old patients who often develops anterior cervical bony spur due to mortality and morbidity resulting from the surgery. The authors have observed a case where even though dysphagia symptoms were not severe, surgery to completely control the symptoms was difficult. In other cases, it was difficult to utilize conservative treatments to treat older patients with dysphagia due to anterior cervical osteophyte. In a third case, after removal of the anterior cervical bony spur and improvement of the dysphagia symptoms, the symptoms reoccurred from reossification and additional surgery was needed. Although surgical treatment is not the preferred approach for patients with dysphagia due to anterior cervical bony spur, it should be considered as one of the methods to improve the symptoms and resolve problems, such as aspiration pneumonia and weight loss resulting from the dysphagia. There is also a need to guide appropriate timing of surgical treatment for patients and a possibility of symptom reoccurrence from reossification after surgery with recommend long term follow-up.

\section{REFERENCES}

1. Freed ML, Freed L, Chatburn RL, Christian M. Electrical stimulation for swallowing disorders caused by stroke. Respir Care 2001; 46: 466-474

2. Utsinger PD, Resnick D, Shapiro R. Diffuse skeletal abnormalities in Forestier disease. Arch Intern Med 1976; 136: 763-768

3. Sobol SM, Rigual NR. Anterolateral extrapharyngeal approach for cervical osteophyte-induced dysphagia. Literature review. Ann Otol Rhinol Larngo 1984; 93: 498-504

4. Kissel P, Youmans JR. Posttraumatic anterior cervical osteophyte and dysphagia: surgical report and literature review. J Spinal Disord 1992; 5: 104-107

5. Evitar E, Harell M. Diffuse idiopathic skeletal hyperostosis with dysphagia (a review). J Laryngol Otol 1987; 101: 627-632

6. Kodama M, Sawada H, Udaka F, Kameyama M, Koyama T. Dysphagia caused by an anterior cervical osteophyte: case report. Neuroradiology 1995; 37: 58-59

7. McGarrah PD, Teller D. Posttraumatic cervical osteophytosis causing progressive dysphagia. South Med J 1997; 90: 858-860

8. Fahrer H, Markwalder T. Dysphagia caused by diffuse idiopathic skeletal hyperostosis. Clin Rheumatol 1988; 7: 117-121

9. Constantoyannis C, Papadas T, Konstantinou D. Diffuse idiopathic skeletal hyperostosis as a cause of progressive dysphagia : a case report. Cases J 2008; 1: 416

10. Miyamoto K, Sugiyama S, Hosoe H, linuma N, Suzuki Y, Shimizu K. Postsurgical recurrence of osteophytes causing dysphagia in patients with diffuse idiopathic skeletal hyperostosis. Eur Spine J 2009; 18: 1652-1658 